\title{
Low Energy Ultrafast Switching in Silicon Wire Waveguides
}

\author{
L.R. Nunes(1a), T.K. Liang(1a), K.S. Abedin(1b),D. Van Thourhout(2), P. Dumon(2), \\ R. Baets(2), H.K. Tsang(3), T. Miyazaki(1b) and M. Tsuchiya(1a)
}

1: NICT - National Institute of Information and Communications Technology, Tokyo, Japan. 1a - Basic and Advanced Research Department $1 \mathrm{~b}$ - Information and Network Systems Department

2: The Chinese University of Hong Kong, Department of Elect. Eng., N.T., Hong Kong

3: Ghent University - IMEC, Department of Information Technology (INTEC), Gent, Belgium email: nunes@nict.go.jp

Abstract $1.9 p$ s optical switching at $40 \mathrm{GHz}$ repetition rate has been successfully demonstrated in submicron-size silicon wire waveguides. Ultrafast operation is achieved by induced optical absorption from two-photon absorption (TPA) process. The device requires very low energy, less than $3 p J$, to accomplish $92 \%$ of modulation depth.

\section{Introduction}

The high refractive index difference $(\Delta>41 \%)$ in Silicon-On-Insulator (SOI) platforms gives rise to the realization of sub-micron order singlemode optical wire waveguides [1]. In such structures, due to the high optical confinement, ultra-high optical intensities can be easily achieved with optical powers typically used in telecommunications. Therefore, the manifestation of nonlinear optical effects at lowenergy is very attractive for the realization of optical devices, especially all-optical switching.

Up to now, most of the reported silicon-based switching devices rely on plasma dispersion effect for their operation principle. To produce the required absorption or phase shift in such devices, excess free carriers are introduced inside the waveguides either by external current injection [2] or optically excitation [3]. Thus the obtained speed, in the order of hundreds of picoseconds, is always limited by the effective carrier lifetime. The use of modulation based on TPA induced free-carrier absorption, which is intrinsically an ultrafast process [4], has also been demonstrated in silicon structures [5-6]. However, the speed was likewise limited in response time by carrier recombination.

In this paper, we demonstrate ultrafast switching based on non-degenerate TPA process [7] in submicron order singlemode optical wire waveguides. 1.9 ps optical switching at $40 \mathrm{GHz}$ repetition rate has been successfully achieved by employing very low energy, less than $3 \mathrm{pJ}$, to accomplish $92 \%$ of modulation depth.

\section{Working principle}

The proposed approach relies on minimizing the free carriers generated inside the waveguide during the switching window, reducing in this way the number of carriers to be subsequently recombined and therefore mitigating the effect of slow recovery, which consequently allows high repetition rate crossabsorption modulation from TPA itself.

The amount of free carriers generated inside the waveguide, during the switching time frame, depends basically on three factors: 1) the peak power of the pulse, 2) the duration of the pulse, and 3) the parameters (physical properties and dimensions) of the waveguides. Since we used ultrashort pulses to achieve high peak power, the amount of free carriers generated during the switching time frame is small. Thus, the effect of slow response time due to free carrier recombination may be minimized by properly fit the pulsewidth and energy of the pump pulses with the parameters of used waveguide.

\section{Experiments}

Figure 1 shows the simplified experimental setup. A semiconductor mode-locked laser generates pump pulses of 1.9ps FWHM pulsewidth at $10 \mathrm{GHz}$ in the wavelength of $1552 \mathrm{~nm}$. The pump pulses are multiplexed up to $40 \mathrm{GHz}$, and then amplified before being combined with the $\mathrm{CW}$ probe signal, which is at the wavelength of $1535.3 \mathrm{~nm}$. The combined signals are coupled into the silicon waveguide. An optical bandpass filter (OBPF) suppresses the pump pulses after the waveguide, allowing only the $40 \mathrm{GHz}$ modulated $\mathrm{CW}$ signal at the output.

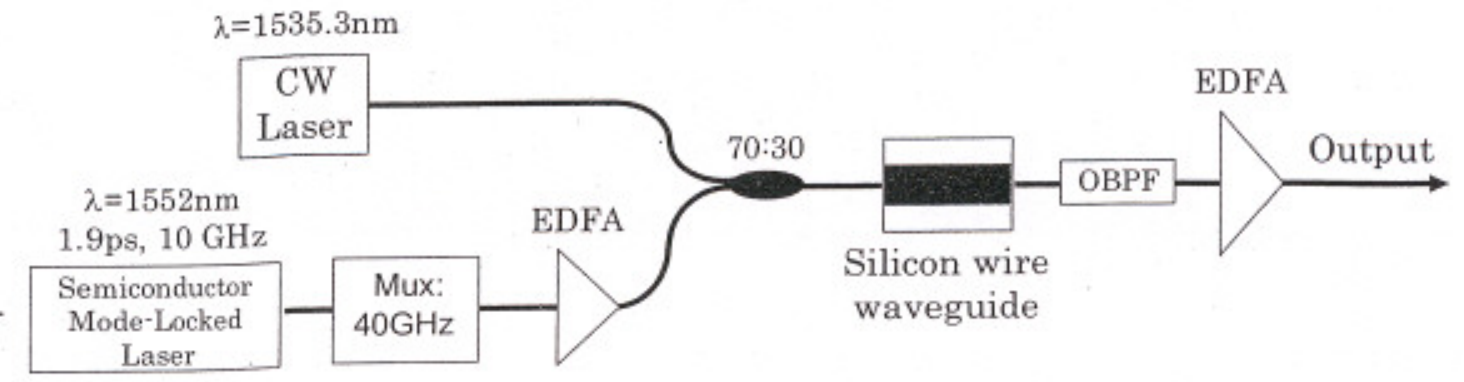

Fig. 1 Simplified Experimental Setup 
The wire waveguides used in the experiments are $10 \mathrm{~mm}$ in length, formed in spiral to fit in a very small foot print area. The waveguide core is formed by a silicon strip measuring $480 \mathrm{~nm}$ in width and $220 \mathrm{~nm}$ in height. The buried oxide layer is $1 \mu \mathrm{m}$. The waveguide propagation loss is $0.5 \mathrm{~dB} / \mathrm{mm}$ and the light is coupled in the waveguides by gratings, which exhibit coupling losses of about $7 \mathrm{~dB}$ per port. The waveguide fabrication process, details on coupling scheme, and characterization of the waveguides are described in [8].

\section{Results and discussions}

Due to the response of the $50 \mathrm{GHz}$ used photodetector, the real modulation depth could not be measured by direct detection and observation on the oscilloscope. Thus, we performed time resolved two color pump-probe measurements. The experiments were performed with both wavelengths with pulsewidth of $1.5 \mathrm{ps}$ at $50 \mathrm{MHz}$, and pump pulse with energy less than $3 \mathrm{pJ}$. The results of the experiments, shown in Fig. 2, indicate that $92 \%$ of modulation depth can be achieved at such low switching energy. The switching time is about the value of the pump pulsewidth. From the same figure, we also confirmed the absence of the slow response due to carrier recombination.

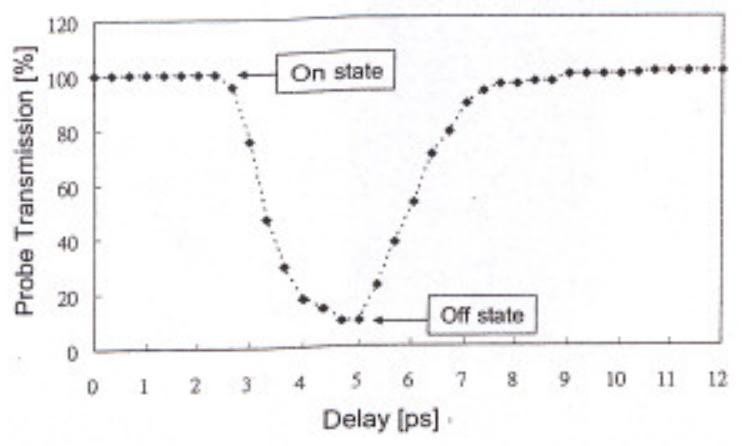

Fig.2. Pump-probe measurements: response time.

We also confirmed the absence of free carrier accumulation at high repetition rates by performing experiments at 10,20 , and $40 \mathrm{GHz}$.

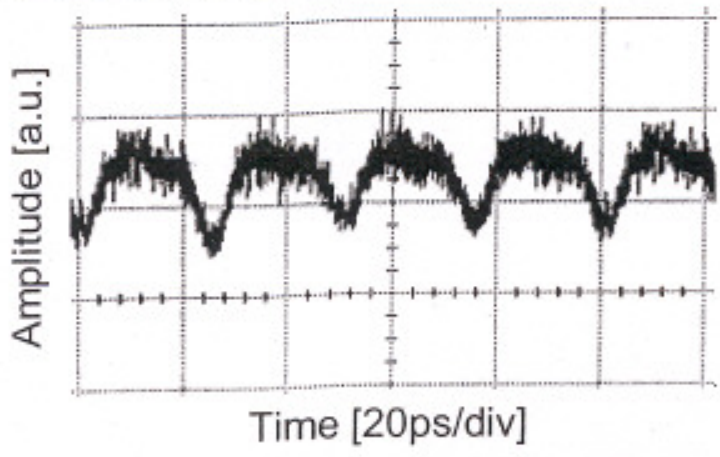

Fig.3 Time domain trace of the $40 \mathrm{GHz}$ Modulated CW after suppression of pump signal.
After passing through the wire waveguide, the CW signal was inversely cross modulated by the pump pulses. The $\mathrm{CW}$ signal coupled into the waveguide was $-5 \mathrm{dBm}$. Figure 3 shows the time domain trace of the $40 \mathrm{GHz}$ modulated $\mathrm{CW}$ light in the form of dark pulses. The shown pulses were broadened to about $13 \mathrm{ps}$ due to the limited bandwidth of the photodetector.

The spectrum of the $40 \mathrm{GHz}$ modulated $\mathrm{CW}$ signal is shown in Fig. 4. The clear spectral lines with $40 \mathrm{GHz}$ spaced from the CW, the center peak, emphasize the efficiency of the modulation.

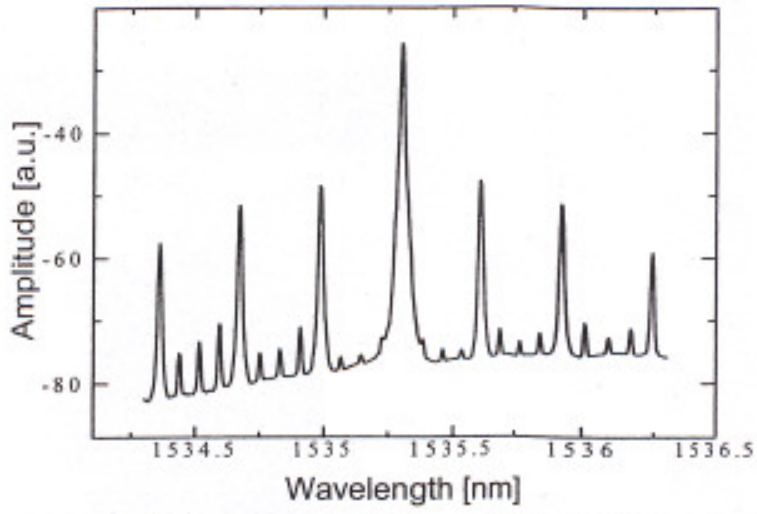

Fig. 4 Optical spectrum of $40 \mathrm{GHz}$ modulated CW signal

Our results show that the effect of slow response time due to free carrier recombination in semiconductors may be reduced by appropriately choosing the pump and probe pulse energies that best fit the parameters of used waveguide. Thus, the proposed scheme can potentially operate at femtosecond switching regime.

As the conversion mechanism was based on TPA process, the signal wavelength can be anywhere between $1200 \mathrm{~nm}$ to beyond $1700 \mathrm{~nm}$ - range at which the sum of pump photon energy and signal photon energy is always higher than the bandgap of silicon.

\section{Conclusion}

We demonstrated ultrafast optical switching in silicon wire waveguides by TPA cross absorption modulation. We confirmed the absence of free carrier accumulation at high bit rates. $92 \%$ of modulation depth was achieved with switching energy less than $3 p J$. Our results show that silicon waveguides have potential applications in ultrafast photonic signal processing.

\section{References}

1 T. Tsuchizawa et al, Quant. Elect. 11, 232 (2005).

2 I. Day et al, OFC'03, 249 (2003).

3 V.R. Almeida et al, Opt. Lett. 29, 2867 (2004).

4 H.K. Tsang et al, Appl. Phys. Lett. 62, 1451 (1993).

5 D. J. Moss, et al. Electron. Lett. 41, 320-321 (2005).

6 H. Fukuda, et al. CLEO/PR'05, CWE1-4,483 (2005).

7 C. Rauscher et al, J. Appl. Phys. 81, 2818 (1997).

8 W. Bogaerts et al, JLT 23, 401-412 (2005). 


\section{1ST EUROPEAN CONFERENCE}
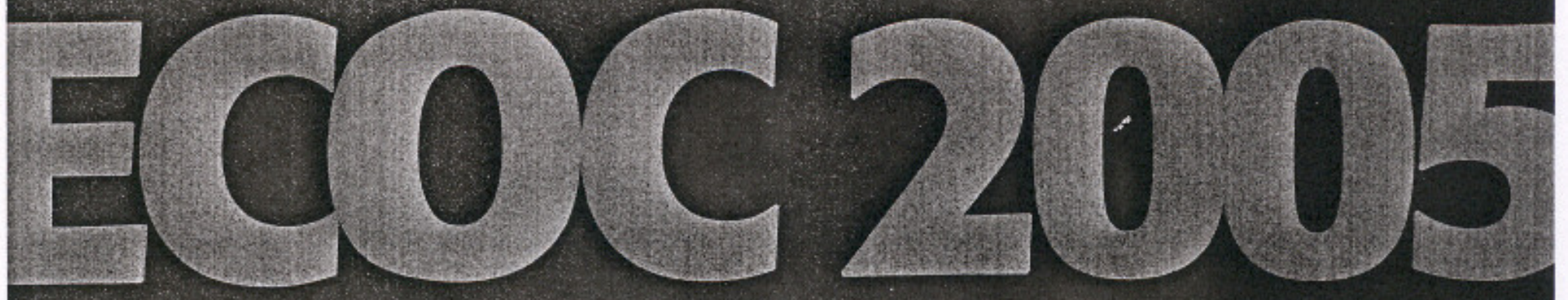

ON OPTICAL COMMUNICATIONS

$315 T$ EUROPEAN

CONFERENCE

ON OPTICAL

COMMUNICATIONS
ORGANISED BY THE IEE

PHOTONICS PROFESSIONAL NETWORK
SECC

GLASGOW

SCOTLAND

$25-29$ SEPTEMBER 2005

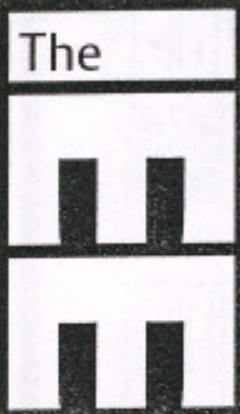

Engincering the future

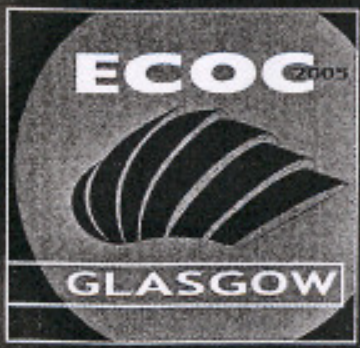

- Volume 6 - Post Deadline 


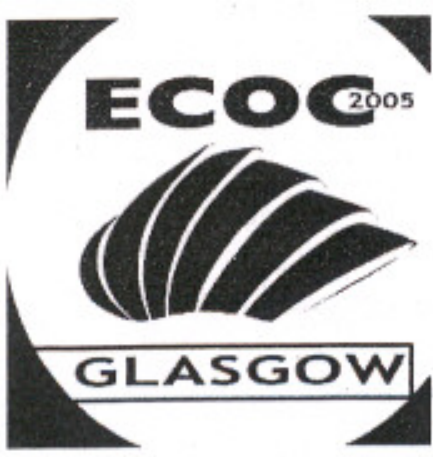

\section{ECOC 2005}

$31^{\text {st }}$ European Conference on

Optical Communication

25-29 September 2005, Glasgow, Scotland

Organised by:

The IEE Photonics Professional Network

In co-operation with:

IEEE/LEOS (Laser and Electro-Optics Society)

EUREL (Convention of National Societies of Electrical Engineers Europe)

OSA (Optical Society of America)

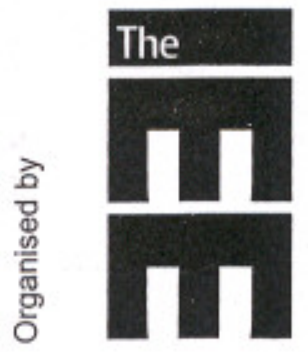

Proceedings

Post-Deadline - Thursday, 29 September 2005 